\title{
On the origin of field O-type stars
}

\author{
E. Schilbach and S. Röser
}

\begin{abstract}
Astronomisches Rechen-Institut, Zentrum für Astronomie der Universität Heidelberg, Mönchhofstraße 12-14, 69120 Heidelberg, Germany

e-mail: [elena;roeser]@ari.uni-heidelberg.de
\end{abstract}

Received 9 April 2008 / Accepted 30 May 2008

\begin{abstract}
Aims. We aim to identify the origins of field O-stars in the nearest 2 to $3 \mathrm{kpc}$ around the Sun using the best presently available kinematic data on O-stars and on young open clusters. We investigate the question of whether the present-day data are consistent with the assumption that $\mathrm{O}$-stars have formed in groups (clusters, associations), or in isolation.

Methods. We apply the epicycle theory to back-trace the orbits of O-type stars and of candidate parent open clusters.

Results. From the 370 O-stars in the "Galactic O star catalog v 2.0" (GOSV2) we have investigated 93 stars classified as field, and found the origin for 73 of them in 48 open clusters younger than 30 Myr. Only for 32 stars or about 9\% of all O-stars from this catalogue is the question of their origin in groups not solved; some of them may have originated in isolation or may have disintegrated the group in which they formed. Fifty percent of the young open clusters (age < $30 \mathrm{Myr}$ ) in the "Catalogue of Open Cluster Data" (COCD) have O-stars as members, or have ejected at least one O-star in the first $10 \mathrm{Myr}$ of their life, or both. During this period the average mass loss from open clusters by ejecting O-stars is found to be 3 to $5 M_{\odot}$ per Myr. We prove that $\zeta$ Pup had its origin in the open cluster Trumpler 10 which it left about $2.5 \mathrm{Myr}$ ago, and that its present-day distance is $300 \mathrm{pc}$ (compared to $440 \mathrm{pc}$ before). The revised distance implies a significant revision of the stellar parameters (a radius of $14 R_{\odot}$, a mass of $22.5 M_{\odot}$, and a luminosity of $\log L / L_{\odot}$ of 5.74) i.e., $\zeta$ Pup is closer, less massive, and less luminous than previously thought. Our findings provide independent estimates of the present-day distances and absolute magnitudes of field O-stars.
\end{abstract}

Key words. stars: early-type - stars: formation - open clusters and associations: general

\section{Introduction}

Do all O-stars form in groups (clusters, associations) as is commonly believed or is the formation of O-stars in isolation possible? This long-standing question can only be answered when the birth-places of all O-stars are discovered. A review of the situation is given in the introduction by Gies (1987) and recently in Zinnecker \& Yorke (2007). Gies (1987) compilied a catalogue of 195 O-stars which he used to infer the first solid statistics about runaway and field O-stars. Recently, a new catalogue of Galactic O-stars (GOSV1 version 1, Maíz-Apellániz et al. (2004); GOSV2 version 2, Sota et al. (2007)) was published. Comprising $370 \mathrm{O}$-stars, the catalogue allows us to re-address the statistics of O-star birth-places. In particular, the GOSV2 catalogue contains a subset of $105 \mathrm{O}$-stars called field, which simply means that they cannot be identified as present or former members of recognised groups. Such "isolated O-stars" are of key interest to decide the question of whether "isolated" massive star formation is possible or not.

Because of the relatively short lifetime (a few million years) near the main sequence, the orbit of an O-type star in the wider solar neighbourhood can, in principle, be followed all the way back in time to the onset of its hydrogen-burning stage. This means that the location of its parent star-forming cloud can be determined. Investigating the area around these parent clouds, one may find other young objects there, e.g. young star clusters or OB-associations.

During the last decade, after the results of the Hipparcos mission became available, nearby OB-associations have been investigated in considerable detail (de Zeeuw et al. 1999). However, not all OB-stars have been found in associations; some are far way from presently-known stellar groups on the sky.

Using the data from ESA's Hipparcos mission, Hoogerwerf et al. (2001) back-traced the orbits of 56 OB-type runaway stars and nine compact objects with distances less than $700 \mathrm{pc}$. They found that at least 21 objects of their sample could be linked back to nearby associations and young open clusters. The authors state that the remaining objects may have originated from distances farther away than $700 \mathrm{pc}$, where the knowledge of parent groups is poor.

Another line of argument has been followed in two papers by de Wit et al. (2004, 2005). In their first paper they investigate the origin of $43 \mathrm{O}$-type field stars from the O-star catalogue by Gies (1987). The authors search the area around these stars for stellar groups in the near-infrared which are possibly hidden in the optical. In their second paper (de Wit et al. 2005) they investigate the same sample kinematically. They excluded as field stars the runaway stars, i.e. those with spatial velocities above the limit of $40 \mathrm{~km} \mathrm{~s}^{-1}$ set by Blaauw (1961) and those at distances larger than $250 \mathrm{pc}$ from the Galactic plane. Combining the results of both papers they claim that not more than $4 \pm 2 \%$ of all O-stars in Gies' catalogue can be called genuine field stars.

The argument by de Wit et al. $(2004,2005)$ is a rather indirect one; they are excluding stars as field without being able to retrace their origin. This is exactly the point where we start our present study. Only if one succeeds in retracing an O-star to a parent group within its past lifetime one can say with certainty that this O-star has originated in a group. Proving or disproving this point is not an easy task given our incomplete knowledge of possible birth-places in the wider neighbourhood of the Sun and 
the uncertainties of the six-dimensional phase space coordinates (position and motion) of candidate stars and candidate clusters and/or associations.

In this paper we test the hypothesis that O-stars, the origin of which is hitherto unknown, may have been ejected from young open clusters (or their protoclusters) during or after the star formation period in the (parental) cluster. For this purpose, we follow the path of stars and clusters back in time in the Galactic potential. In the next section we present the underlying observations, then we describe the method and its application. Sect. 4 is a presentation and a discussion of the results. In Sect. 5 we consider a few selected examples of stars with the adopted solutions, and in Sect. 6 we briefly discuss the stars for which we did not find an acceptable solution. A summary concludes the paper.

\section{Observational material}

For the back-tracing of stellar and cluster orbits we use the most homogeneous and accurate data of all 6 parameters of phase space available at present. We took the positions and proper motions from the recently completed PPMX catalogue (Röser et al. 2008), and the radial velocities from the CRVAD-2 (Kharchenko et al. 2007). The major sources of specific information on open clusters and O-type stars were the catalogues by Kharchenko et al. (2005a,b) and Sota et al. (2007), respectively.

\subsection{Open clusters}

The Catalogue of Open Cluster Data (COCD) and its Extension (Kharchenko et al. 2005a,b) includes 641 open clusters and 9 cluster-like associations identified in the ASCC-2.5 catalogue (Kharchenko 2001). For each cluster the membership was determined using spatial, kinematic, and photometric criteria (Kharchenko et al. 2004). A homogeneous set of cluster parameters was derived by applying a uniform technique. The nine associations are included in the COCD because of their compact appearance on the sky and small dispersion in proper motion space, so that they can be treated with our standard membership selection procedure. The COCD contains the celestial position of a cluster, its distance to the Sun, reddening, age, angular size, proper motions, and, if available, radial velocity. Recently, the parameter set was supplemented by tidal radii and masses (Piskunov et al. 2008a). The completeness of the cluster sample is mainly defined by the limiting magnitude of $V \approx 11.5$ of the ASCC-2.5. Therefore, even nearby embedded clusters could be missing in the sample if their members are fainter than $V \approx 11.5$ in the optical. Nevertheless, for "classical" open clusters, i.e. when the bulk of the placental matter is removed and clusters become visible in the optical spectral range, the sample was found to be complete up to a distance of about 850 pc (Piskunov et al. 2006).

In the context of this paper, however, we are not interested in the full sample, but in a sub-sample of young clusters. As potential candidates for parent groups we considered 161 clusters (including 9 associations) younger than $30 \mathrm{Myr}$, and having measured radial velocities. Since absolutely bright stars are still present in these clusters, this subset is volume limited to about $2 \mathrm{kpc}$ (Piskunov et al. 2006), or to a distance modulus $\left(V-M_{V}\right) \approx 13$ (Schilbach et al. 2006) when extinction is taken into account. Except for proper motions, the cluster data for each

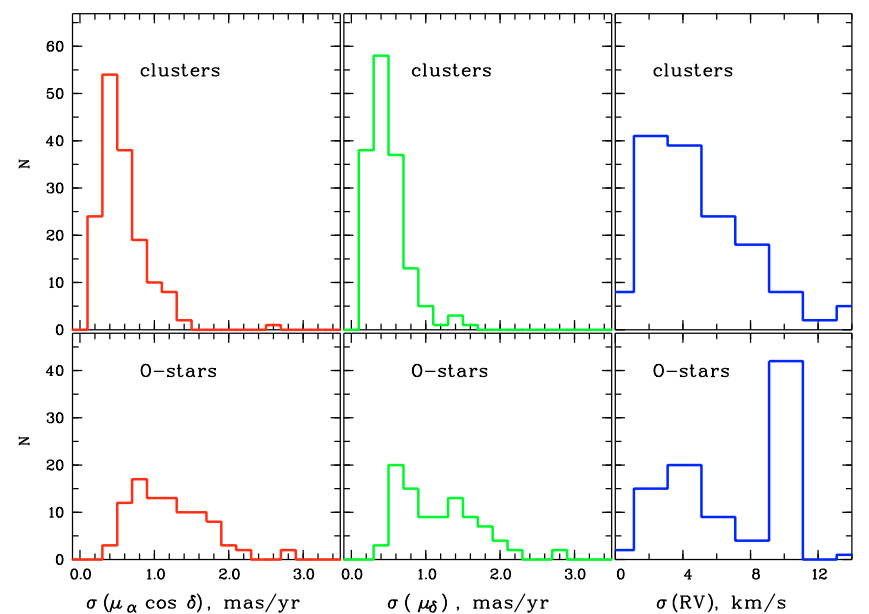

Fig. 1. The distribution of the rms errors of proper motions $\mu_{\alpha} \cos \delta$ (left), $\mu_{\delta}$ (middle), and of radial velocities $R V$ (right). Upper panels show the distributions for the 161 young open clusters, lower panels for the 93 field O-stars. The peak at $10 \mathrm{~km} \mathrm{~s}^{-1}$ in $\sigma_{R V}$ of O-stars represents stars for which no information on the rms error of the radial velocity is given in the literature.

cluster (i.e., coordinates of the cluster centre, distance, and radial velocity) were taken from the COCD. The mean proper motions were recomputed from the PPMX data and the membership information given in Kharchenko et al. (2004). In the upper panels of Fig. 1, we show histograms of the mean errors of the kinematic data for this sub-sample of young clusters.

\subsection{O-type stars}

The sample of O-type stars for this paper was taken from the second version GOSV2 (Sota et al. 2007) of the "Galactic O star catalog" by Maíz-Apellániz et al. (2004), with 370 entries. The catalogue is expected to be complete for O-stars brighter than $V=8$ but it includes many fainter stars, too. For each star, the catalogue delivers spectral classification, photometric and astrometric data, and further information such as multiplicity and membership in known associations. Among 370 stars, 105 stars are classified as field stars or field runaways from unknown parent groups. These are the stars we were interested in.

Again, we took the positions and proper motions from the PPMX catalogue. Doing so, we not only benefitted from accurate proper motions but also kept proper motions of clusters and O-stars in the same system. Radial velocities came from CRVAD-2. Though CRVAD-2 presents data for 55000 stars, radial velocities are available for only 93 O-stars of our list. Unfortunately, for 41 stars, no information on the rms errors of radial velocities is available, so we assumed them to be $\pm 10 \mathrm{~km} \mathrm{~s}^{-1}$. The histograms of the mean errors of kinematic data for the final sample of O-stars are shown in the lower panels of Fig. 1. As expected, the accuracy of the data for clusters is, on average, higher than for O-stars. For clusters, the median of the $r m s$ errors of $\mu_{\alpha} \cos \delta, \mu_{\delta}$ and $R V$ are 0.5 mas/yr, 0.4 mas/yr and $3.4 \mathrm{~km} \mathrm{~s}^{-1}$, respectively. For O-stars, the corresponding numbers are $1.2 \mathrm{mas} / \mathrm{yr}, 1.0 \mathrm{mas} / \mathrm{yr}$ and $7.5 \mathrm{~km} \mathrm{~s}^{-1}$.

The distances of O-stars are more of a problem. The GOSV2 gives Hipparcos (ESA 1997) parallaxes for all stars for which these are available. van Leeuwen (2007) published the new reduction of the Hipparcos observations. Due to a sophisticated modelling of the satellite's attitude, van Leeuwen (2007) could considerably improve the trigonometric parallaxes of stars 
brighter than about 8th visual magnitude. However, only five Ostars of our sample had Hipparcos parallaxes with an accuracy better than $30 \%$.

In order to obtain distances for all stars, we had to use the methods of distance estimates based on the spectroscopic and photometric data. To derive spectroscopic distances $d_{\text {sp }}$ from the well-known relation

$$
\log d_{\mathrm{sp}}=0.2\left[V-M_{V}+5-3.1\left((B-V)-(B-V)_{0}\right)\right],
$$

we took the observed $B$ and $V$ magnitudes and the spectral classification from the GOSV2 catalogue, and converted spectral type into absolute magnitude $M_{V}$ and $(B-V)_{0}$ according to Schmidt-Kaler (1982). However, the spectral classification of Ostars is neither straightforward nor unambiguous. For example, for a relatively bright star, HD $135240(V=5.08)$, one finds a spectral type and luminosity class of O 7.5 III in the GOSV2 catalogue and $\mathrm{O} 8.5 \mathrm{~V}$ in SIMBAD. This difference in spectral classification leads to an uncertainty of about $1 \mathrm{mag}$ in distance modulus. The second source of uncertainty arises from the $M_{V^{-}}$ spectral-type calibration which can introduce systematic effects of up to 1 mag (e.g., see Walborn 2002). Moreover, the calibration of O-stars shows a large scatter which may be intrinsic to the stars themselves (e.g., Conti et al. 1983; Garmany \& Stencel 1992). We conclude that the spectroscopic distance moduli may be uncertain by up to $2 \mathrm{mag}$, and the distance of a field $\mathrm{O}$-star is the most inaccurate input parameter in the back-tracing of stellar and cluster orbits.

\section{The back-tracing method}

\subsection{The epicycle approach}

For the re-tracing of the stars and open clusters we followed the approach used by Fuchs et al. (2006) who adopted the epicyclic equations of motion as given by Lindblad (1959):

$$
\begin{aligned}
\xi(t)= & \xi(0)+\frac{v(0)}{2 B}[1-\cos (\kappa t)]+\frac{u(0)}{\sin (\kappa t)}, \\
\eta(t)= & \eta(0)+2 A\left[\xi(0)+\frac{v(0)}{2 B}\right] t \\
& -\frac{\Omega_{0}}{B \kappa} v(0) \sin (\kappa t)+\frac{2 \Omega_{0}}{\kappa^{2}} u(0)[1-\cos (\kappa t)], \\
\zeta(t)= & \zeta(0) \cos (v t)+\frac{w(0)}{v} \sin (v t), \\
u(t)= & u(0) \cos (\kappa t)+\frac{\kappa}{2 B} v(0) \sin (\kappa t), \\
v(t)= & -\frac{2 B}{\kappa} u(0) \sin (\kappa t)+v(0) \cos (\kappa t), \\
w(t)= & w(0) \cos (v t)-\zeta(0) v \sin (v t)
\end{aligned}
$$

where $A$ and $B$ are Oort's constants, $\Omega_{0}$ is the angular velocity of the Galactic rotation of the local standard of rest (LSR), $\kappa$ and $v$ are the epicycle frequency and the vertical oscillation frequency, respectively. We assumed a flat rotation curve $A=-B=\Omega_{0} / 2$, where $\Omega_{0}=25.9 \mathrm{~km} \mathrm{~s}^{-1} \mathrm{kpc}^{-1}$ at the Galactocentric radius of the LSR $r_{0}=8.5 \mathrm{kpc}$. We also adopted the same values as Fuchs et al. (2006) for the parameters $\kappa=39.0 \mathrm{~km} \mathrm{~s}^{-1} \mathrm{kpc}^{-1}$ and $v=$ $74 \mathrm{~km} \mathrm{~s}^{-1} \mathrm{kpc}^{-1}$.

Equations (2) and (3) describe the motion of a particle in a non-inertial coordinate system centred at a fiducial point at a Galactocentric radius $r_{0}$ (at $Z=0$ ) from the Galactic centre, for which the transformation from the Cartesian Galactic coordinates $X, Y, Z$ into $\xi, \eta, \zeta$ is given by

$$
\begin{aligned}
\xi & =r_{0}-r \\
\eta & =r_{0} \times \arctan (Y / r) \\
\zeta & =Z
\end{aligned}
$$

with $r=\sqrt{\left(r_{0}-X\right)^{2}+Y^{2}}$. Similarly, the velocity components $U, V, W$ of the peculiar space velocity (after correcting for solar motion and Galactic rotation) are transformed into $u, v, w$ via

$$
\begin{aligned}
u & =U \frac{r_{0}-X}{r}-V \frac{Y}{r}, \\
v & =U \frac{Y}{r}+V \frac{r_{0}-X}{r}, \\
w & =W .
\end{aligned}
$$

As we are only interested in the relative location and velocity of a star with respect to a candidate parent cluster, we chose $r_{0}$ close to the Galactocentric radius of the candidate cluster in each case, and the flat rotation curve gave $\Omega_{0}=220 / r_{0} \mathrm{~km} \mathrm{~s}^{-1} \mathrm{kpc}^{-1}$. The epicycle and the oscillation frequencies have been assumed to be constant. This extends the validity of the approach, as the initial $\xi, \eta$ are small for star and cluster, even at larger distances from the Sun, provided that $r_{\text {star }}-r_{0} \ll r_{0}$, and $v \ll r_{0} \Omega_{0}$. We checked that the requirements were fulfilled for our solutions.

\subsection{Variation of the initial conditions and selection of the solution}

As a starting point of the backward computations, we took the positions, proper motions, radial velocities of stars and clusters at their nominal values from the sources described in Sect. 2. However, taking into account the quality of the input data, we allowed variations of initial conditions for O-stars in Eqs. (2) and (3) within the given error budget. We varied the nominal values of their proper motions and radial velocities between $-2.5 \sigma$ and $+2.5 \sigma$ in $0.5 \sigma$ steps, and the distance moduli between $\left[\left(V-M_{V}\right)-2\right]$ and $\left[\left(V-M_{V}\right)+2\right]$ in steps of 0.02 mag. Initial conditions for the clusters were not varied.

The orbits were traced back in time over $11 \mathrm{Myr}$ with a step of 0.05 million years. At each time step, the relative distance between each star and each cluster was determined. For the rest of the paper we adopt the following terminology. We speak of an encounter, if, going backward in time, the distance between star and cluster (centre) was less than $10 \mathrm{pc}$. This is called an acceptable solution. The encounter time, $t_{\mathrm{enc}}$, is the time before present when an encounter occurred, and the encounter distance, $d_{\text {enc }}$, is the distance between star and cluster centre at $t_{\text {enc }}$. Note, however, that the actual physical process is an ejection of a star from its cluster counterpart. This ejection occurred at time $t_{\mathrm{enc}}$, when the star was at a distance $d_{\text {enc }}$ from the cluster centre.

For each acceptable solution, we computed a probability $p_{\text {kin }}$ describing how well the proper motions and radial velocities (actually used in the solution) suit the nominal values of the kinematic parameters of given star and cluster:

$p_{\text {kin }}=\exp \left\{-\frac{1}{6}\left[\left(\frac{\Delta \mu_{\alpha, \mathrm{s}}}{\Sigma_{\mu_{\alpha, \mathrm{s}, \mathrm{c}}}}\right)^{2}+\left(\frac{\Delta \mu_{\delta, \mathrm{s}}}{\Sigma_{\mu_{\delta, \mathrm{s}, \mathrm{c}}}}\right)^{2}+\left(\frac{\Delta R V_{\mathrm{s}}}{\Sigma_{R V, \mathrm{~s}, \mathrm{c}}}\right)^{2}\right]\right\}$.

Here $\Delta \mu_{\alpha, \mathrm{s}}, \Delta \mu_{\delta, \mathrm{s}}$, and $\Delta R V_{\mathrm{s}}$ are differences between the applied and the nominal values of the corresponding velocity components of the star, and $\left(\Sigma_{\mathrm{vc}, \mathrm{s}, \mathrm{c}}\right)^{2}=\sigma_{\mathrm{vc}, \mathrm{s}}^{2}+\sigma_{\mathrm{vc}, \mathrm{c}}^{2}$ where $\sigma_{\mathrm{vc}, \mathrm{s}}$ and $\sigma_{\mathrm{vc}, \mathrm{c}}$ are the rms errors of the corresponding velocity component 


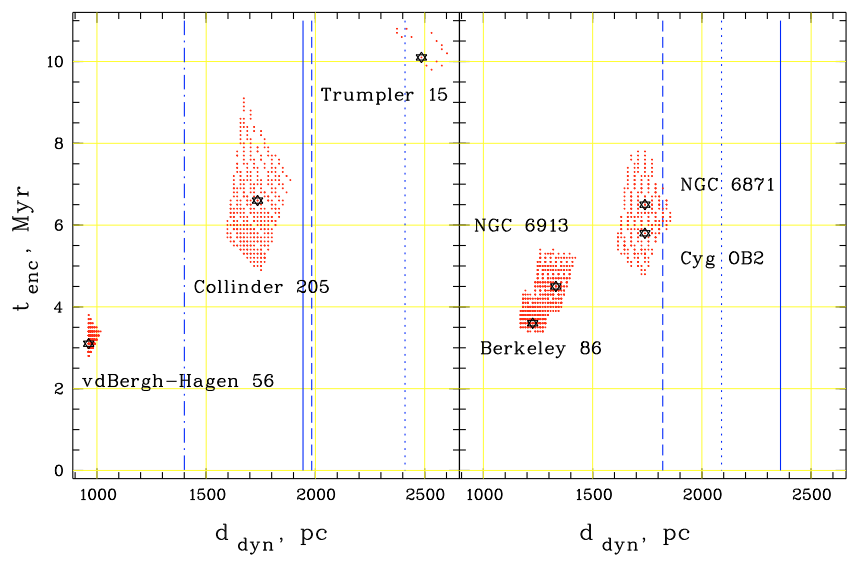

Fig. 2. Dynamic distance versus back-tracing time for HD 75222 (left panel) and HD 201345 (right panel). Dots indicate all acceptable solutions, stars show the best solutions for an individual starcluster combination. Vertical lines mark different distance estimates for these stars: the solid line is for a spectro-photometric distance from Neckel et al. (1980), the dotted and dashed lines are for spectroscopic distances based on spectral types from Sota et al. (2007) and $M_{V}$-spectral-type calibrations from Schmidt-Kaler (1982) and Garmany \& Stencel (1992), respectively. The dashed-dotted line is a distance estimate taking the spectral type from Houk \& Swift (1999) and calibrations from Schmidt-Kaler (1982).

of the star and cluster under consideration. If $\Delta$ is smaller than $\Sigma$ in each of three velocity components, then the corresponding solutions has a kinematic probability $p_{\text {kin }}>0.606$.

This procedure yielded a three-parameter set i.e., encounter time $t_{\text {enc }}$, present-day (or dynamic) distance $d_{\text {dyn }}$ of an O-star from the Sun, and kinematic probability $p_{\text {kin }}$ of acceptable solutions for the encounter distance $d_{\text {enc }}$. In the next step, we selected $10 \%$ of solutions with the largest $p_{\text {kin }}$ and gave the highest priority to the solution providing the smallest $d_{\mathrm{enc}}$. If, for a given $\mathrm{O}$-star there were solutions encountering more than one cluster, we took into account the available information on distance estimates for this star (e.g., spectroscopic distance, spectrophotometric parallax, Hipparcos parallax) in making the decision.

In Fig. 2 we give two examples of such sets of solutions. The left panel of Fig. 2 shows the case of HD 75222, the right panel is for HD 201345. For HD 75222 we obtain three sets of acceptable solutions depending on the present-day distance $d_{\text {dyn }}$ of the star from the Sun. Nevertheless, the solution including vdBergh-Hagen 56 as a cluster that the star encountered about 3 Myr ago can be rejected due to poor compatibility with spectroscopic and spectro-photometric distance estimates. The solution that includes Trumpler 15 fits a distance estimate based on $M_{V}$-spectral-type calibrations from Schmidt-Kaler (1982) but needs strong variations in all three kinematic parameters, so even for the best solution the kinematic probability is smaller than 0.2 . On the other hand, the solution with Collinder 205 coincides better with the other distance estimates available for HD 75222, and it is quite a stable one. We conclude: assuming a presentday distance $d_{\text {dyn }}=1735 \mathrm{pc}$, HD 75222 was ejected from the young cluster Collinder 205 at $d_{\mathrm{enc}} \approx 1 \mathrm{pc}$ about $6.6 \mathrm{Myr}$ ago. The kinematic probability of this solution is 0.96 .

The case of HD 201345 is less clear. There are four sets of acceptable solutions. Again, encounters with NGC 6913 and Berkeley 86 can be rejected since they assume a present-day distance of the star of about $1.2 \mathrm{kpc}$, which is too small with respect to the spectroscopic distance estimates. The solutions

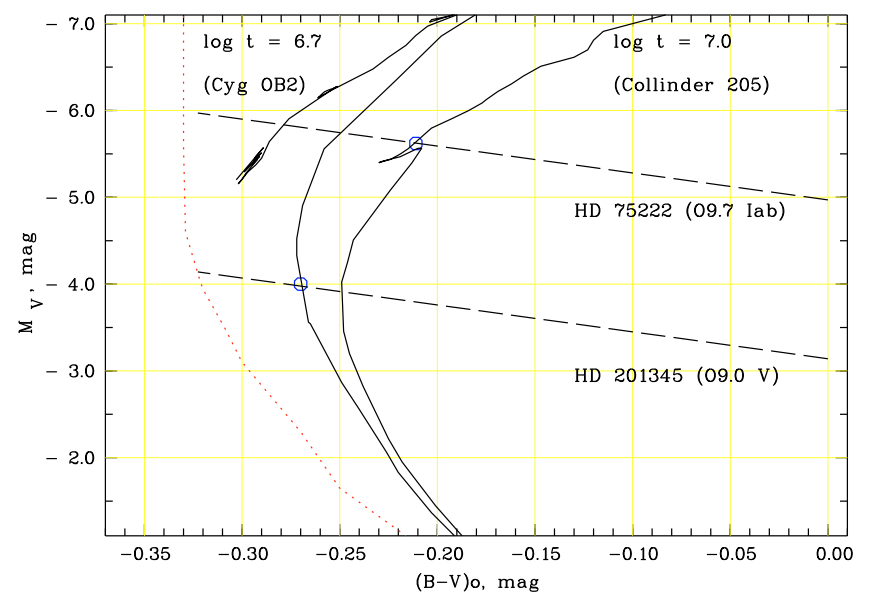

Fig. 3. The colour-magnitude diagram $\left((B-V)_{0}, M_{V}\right)$ of the acceptable solutions for HD 75222 and HD 201345. The dashed lines show $\left(M_{V}-\right.$ $\left.3.1(B-V)_{0}\right)$ lines based on Eq. (1) and dynamical distances $d_{\mathrm{dyn}}$ derived from the back-tracing procedure for HD 75222 and HD 201345. The dotted line stands for the ZAMS, whereas solid lines indicate isochrones of Collinder 205 and Cyg OB2 found to be the hosts of HD 75222 and HD 201345, respectively. Small circles mark the absolute magnitude adopted for HD $75222\left(M_{V}=-5.6\right)$ and HD $201345\left(M_{V}=-4.0\right)$.

with NGC 6871 and Cyg OB2 as counterparts assume both a present-day distance of $d_{\text {dyn }}=1740$ pc for HD 201345 and fit much better the spectroscopic distance estimates. With a kinematic probability $p_{\text {kin }}=0.99$ the star was ejected from Cyg OB2 at $d_{\text {enc }}=1.5 \mathrm{pc}$ about $5.8 \mathrm{Myr}$ ago. For the solution with NGC 6871 we obtained $d_{\text {enc }}=1 \mathrm{pc}, t_{\mathrm{enc}}=6.5 \mathrm{Myr}, p_{\text {kin }}=0.85$. Since the kinematic probability of the solution with Cyg OB2 is higher, we selected Cyg OB2 as the most probable host of HD 201345. However, the solution with NGC 6871 cannot be rejected completely. We discuss this case below in Sect. 5 which is devoted to the results on individual stars.

For each star with a present-day distance $d_{\text {dyn }}$ determined, we can estimate its absolute magnitude $M_{V}$ using Eq. (1) which directly provides a relation $\left(M_{V}-3.1(B-V)_{0}\right)$. Assuming that the adopted extinction law is correct and the intrinsic colour $(B-V)_{0}$ of an O-star is between $-0.33 \mathrm{mag}$ and $-0.13 \mathrm{mag}$, we obtain a maximum variation of about 0.6 mag for its absolute magnitude $M_{V}$. The limit of $-0.33 \mathrm{mag}$ is defined by the location of the ZAMS, whereas the limit -0.13 mag corresponds to spectral types from B7 V to B3 I (see Schmidt-Kaler 1982) and it was chosen not to exclude O-stars. Assuming further that the star is "genetically" related to its counterpart-cluster, we chose the crossing point between the line $\left(M_{V}-3.1(B-V)_{0}\right)$ for the star and the isochrone corresponding to the cluster age to be the absolute magnitude $M_{V}$ of the star. For illustration, we show the corresponding $\left((B-V)_{0}, M_{V}\right)$ plot in Fig. 3 for HD 75222 and HD 201345. The adopted absolute magnitudes $M_{V}$ are -5.6 and -4.0 for HD 75222 and HD 201345, respectively.

\section{Results}

For 73 out of 93 O-stars considered, we found acceptable solutions indicating that the present-day data are consistent with the assumption that these O-stars had encountered (actually, are ejected from) young open clusters during the past $10 \mathrm{Myr}$. The essential results of the back-tracing calculations are compiled in Table 2. For each of the 73 O-stars we give: HD identification (Col. 1), its spectral classification taken from the GOSV2 catalogue (2), name and age (Kharchenko et al. 2005a,b) of the 


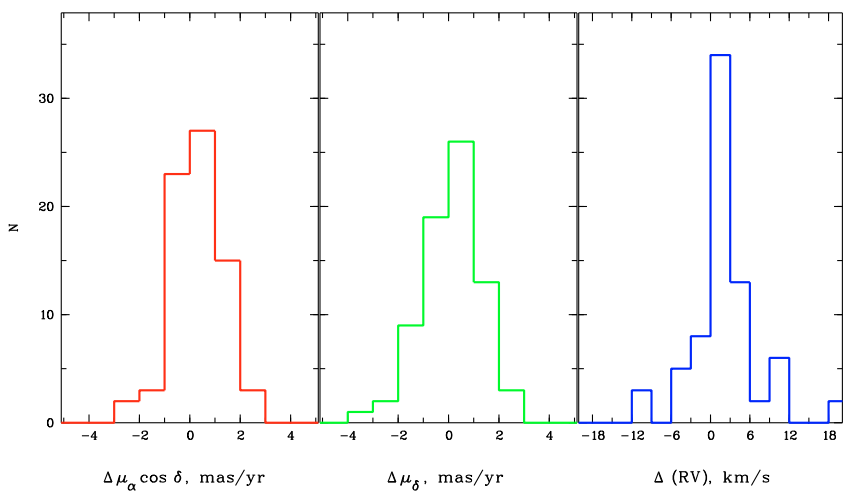

Fig. 4. Distribution of off-sets introduced in proper motions $\mu_{\alpha} \cos \delta$ (left), $\mu_{\delta}$ (middle), and in radial velocities $R V$ (right) of the O-stars with acceptable solutions.

probable counterpart cluster $(3,4)$, the dynamical distance of the star $d_{\text {dyn }}(5)$. Column 6 gives the time $t_{\text {enc }}$, i.e. the time before present when the star was ejected. Columns 7 and 8 are the distance $d_{\text {enc }}$ and the relative velocity $\Delta V e l_{\text {enc }}$ between the star and the cluster at $t_{\text {enc }}$. Column 9 contains the star's absolute magnitude and its upper and lower limits estimated from $d_{\mathrm{dyn}}$ (see the end of Sect. 3.2), and Col. 10 gives the kinematic probability $p_{\text {kin }}$ of the solution.

Except in one case, the kinematic probability $p_{\text {kin }}$ is always larger than 0.5 . This means that in units of rms errors, only small variations of the kinematic parameters were needed to obtain an acceptable solution for the majority of stars. The distributions of off-sets in the sense (used parameter - nominal parameter) are shown in Fig. 4 for proper motions and radial velocities of the 73 stars with acceptable solutions. The distributions do not indicate any anomalies, so we conclude that the results of the back-tracing calculations can be used to understand where the field O-stars came from and explain their present location.

Of 161 young open clusters in our sample, only 48 clusters were "hosts" of field O-stars. Only two clusters are older than 20 Myr. Twelve clusters ejected two O-stars each, two clusters (Cyg OB2, and ASCC 8) had three ejections in the past, and three clusters (Trumpler 14, Loden 821 and ASCC 9) even four ejections. Three O-stars turned out to be members of the newly detected open clusters ASCC 45 and ASCC 79 (Kharchenko et al. 2005b). Since their origin seems to be clear, they are excluded from the statistics presented below.

In Fig. 5 we show the ages of the clusters versus encounter time $t_{\text {enc }}$. Close to the bisector in this figure, one expects to find $\mathrm{O}$-stars that left the cluster birth-places just before, during or just after the time the clusters were forming. In this sense they were members of protoclusters but did not become actual cluster members; they were formed in the same regions where the corresponding clusters originated. On the other hand, there is a number of $\mathrm{O}$-stars with $t_{\text {enc }}$ significantly less than the ages $t_{\mathrm{ocl}}$ of their counterparts. For these stars, we assume that they were ejected from the already formed cluster at an early stage of the cluster's life, either due to internal evolutionary processes in the cluster itself, due to binary evolution or due to external disturbing forces (see e.g. Kroupa \& Boily 2002; Zinnecker 2003). We have marked these candidates with crosses in Fig. 5 when $t_{\mathrm{enc}}<0.5 \times t_{\mathrm{ocl}}$. This criterion is related to the accuracy of the age estimates of open clusters in our sample which is found to be about $\sigma_{\log t}=0.20 \ldots 0.25$ (Kharchenko et al. 2005a). This determination of cluster ages is based on the Padova isochrones (Girardi et al. 2002) which have a lower limit

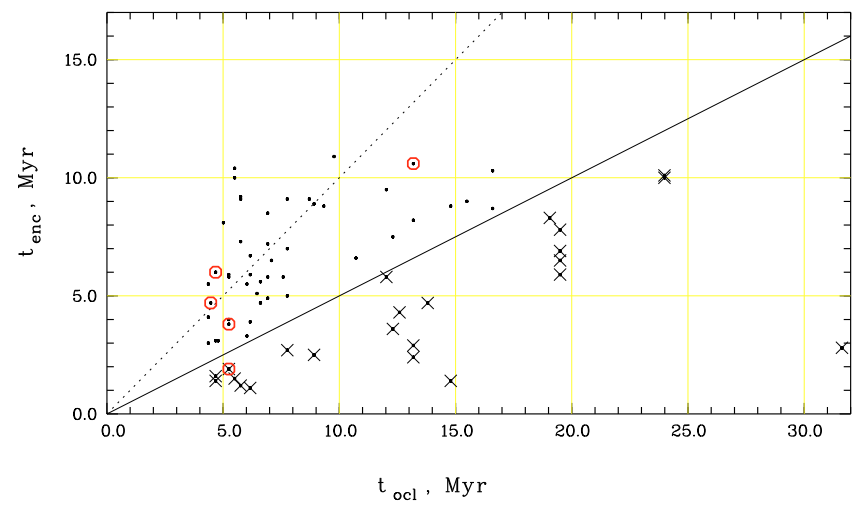

Fig. 5. Cluster age $t_{\mathrm{ocl}}$ versus encounter (ejection) time $t_{\mathrm{enc}}$. Dots represent the $70 \mathrm{O}$-stars with acceptable solutions from the back-tracing procedure. Crosses mark $23 \mathrm{O}$-stars that were possible cluster members at the beginning of their life but, later, were ejected from the parent clusters. The solid line $t_{\mathrm{enc}}=0.5 \times t_{\mathrm{ocl}}$ separates them from the other O-stars; the dotted line is the bisector. The red open circles indicate O-stars with velocities higher than $80 \mathrm{~km} \mathrm{~s}^{-1}$ with respect to their cluster counterparts at the moment of the closest approach (ejection).

at $\log t_{\mathrm{ocl}}=6.6$. Therefore, the ages of the youngest clusters may be somewhat overestimated. Taking this into account, the portion of ejected $\mathrm{O}$-stars from already formed clusters should be about 25-35\%. The majority of O-stars, however, was ejected from the star formation region during the protocluster phase. The fact that we do presently observe the outcome of this latter scenario as an "isolated" O-star and a surviving open cluster may indicate that cluster disruption by O-stars in early stages is possibly less effective than assumed by e.g. Lada \& Lada (2003).

From the study of the initial mass function of Galactic open clusters, Piskunov et al. (2008b) found that a typical cluster loses about $60-80 \%$ of its initial mass during the first $260 \mathrm{Myr}$ of its evolution. The average mass loss rate determined by Piskunov et al. (2008b) ranges from 3 to $14 M_{\odot} / \mathrm{Myr}$ which includes mass loss due to stellar and dynamical evolution. From the number of ejected O-stars, the number of parent clusters, and the distribution of their encounter times $t_{\text {enc }}$, we can roughly estimate the average mass loss of a cluster caused by the ejection of O-stars alone. Assuming a typical mass of an O-star of $20 M_{\odot}$, we obtain the average mass loss rate of a cluster due to ejected Ostars from about $5 M_{\odot} / \mathrm{Myr}$ if $t_{\mathrm{enc}}<5 \mathrm{Myr}$ to $3 M_{\odot} / \mathrm{Myr}$ if $5 \mathrm{Myr}<t_{\mathrm{enc}}<11 \mathrm{Myr}$. Though the statistics is rather poor, the result agrees well with the estimation by Piskunov et al. (2008b) and underlines the importance of the contribution of ejected O-stars to the general mass loss of open clusters in the first 10 Myr of their life.

At the moment of ejection, the relative velocities of former cluster members with respect to their parent clusters are rather moderate, with a median at $\Delta V e l \approx 45 \mathrm{~km} \mathrm{~s}^{-1}$. This does not change significantly if we consider all 70 O-stars. In Fig. 6 we show the histogram of relative velocities $\Delta \mathrm{Vel}$ for all O-stars with acceptable solutions and for the 23 stars that probably were cluster members in the past. Of five stars with $\Delta V e l$ higher than $80 \mathrm{~km} \mathrm{~s}^{-1}$, there is only one that was a former cluster member. The other four probably belonged to protoclusters and were ejected from the regions during cluster formation.

As described in Sect. 4, our back-tracing procedure allows us to determine the present-day, or dynamical distances $d_{\text {dyn }}$ of O-stars. This gives us estimates of their absolute magnitudes $M_{V \text {,dyn }}$. In Fig. 7 we show the distribution of differences $\Delta M_{V}=M_{V, \text { st }}-M_{V, \text { dyn }}$ to the standard calibrations 


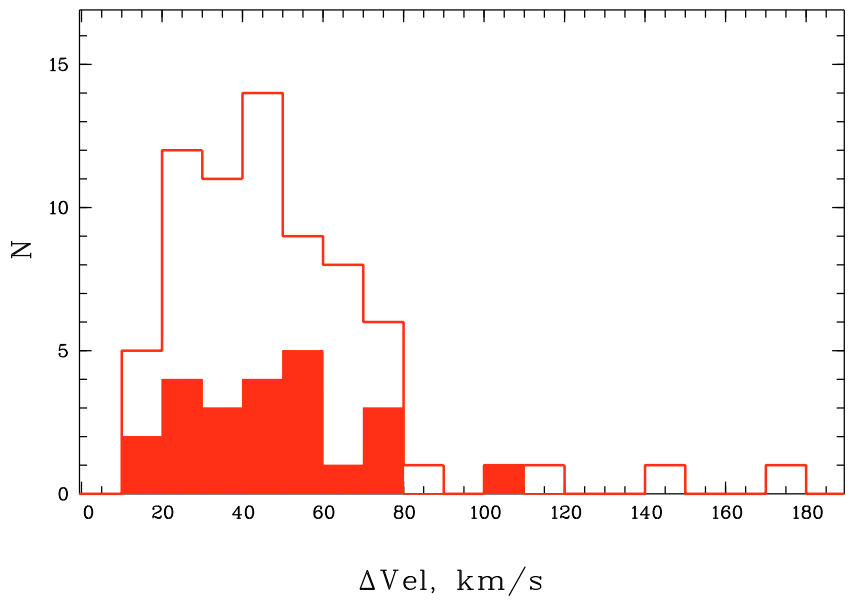

Fig. 6. Distribution of relative velocities of O-stars with respect to their cluster counterparts at the moment of the closest approach (ejection). The filled histogram shows former cluster members ejected from their hosts.

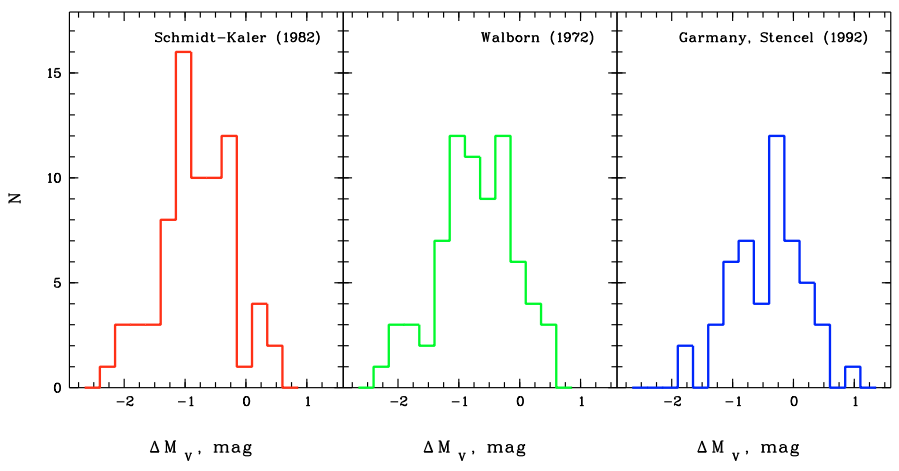

Fig. 7. Distribution of differences in the absolute magnitudes of field O-stars derived from our dynamical $d_{\text {dyn }}$ distances versus the absolute magnitudes according to the calibrations by Schmidt-Kaler (1982), Walborn (1972), and Garmany \& Stencel (1992), respectively. On average, our $d_{\text {dyn }}$ lead to systematically lower $M_{V}$ than the previous calibrations.

from Schmidt-Kaler (1982), Walborn (1972), and Garmany \& Stencel (1992). At first glance this picture is intriguing, but the calibrations of absolute magnitudes of O-stars date back to the pre-Hipparcos era. After Hipparcos it has become possible to carry out a re-calibration, although more for early B-type stars than for the O-stars themselves. Utilizing the measurements from Hipparcos, Kaltcheva (1999) found $M_{V, \text { st }}-M_{V, \text { Hipp }}=$ $-0.85 \pm 0.12$ for 44 B0-B3 III, IV stars. This number coincides well with our findings for the median of $\Delta M_{V}=-0.84,-0.72$ or -0.36 depending on the different calibrations (see Fig. 7). As we will show below, $\zeta$ Pup, the only O-star with a highly significant parallax in van Leeuwen (2007), has an $M_{V, \text { st }}-M_{V, \text { Hipp }}=-1.34$.

\section{Individual stars}

In the following we discuss the results for a few selected stars in more detail. These include the case of $\zeta$ Pup, the closest O-star to the Sun, the case of a common origin of $4 \mathrm{O}$-stars, and finally the case of O-stars presently at large distances from the Galactic plane.
Table 1. $\zeta$ Puppis: distances, distance moduli $V-M_{V}$, and derived differences in absolute magnitude $\mathrm{M}_{V}$ with respect to the new Hipparcos parallax (van Leeuwen 2007).

\begin{tabular}{cccc}
\hline \hline Distance, pc & $V-M_{V}$ & $\Delta M_{V}$ & Sources for distance \\
\hline 333 & 7.60 & 0.00 & van Leeuwen (2007) \\
429 & 8.16 & -0.56 & ESA (1997) \\
615 & 8.94 & -1.34 & Spectral type O4 I \\
300 & 7.36 & +0.24 & this paper \\
\hline
\end{tabular}

\section{1. $\zeta$ Pup (HD 66811)}

$\zeta$ Pup is an important benchmark for the astrophysical characteristics of massive stars. Spectroscopically it is classified as O4 I according to Sota et al. (2007), and its trigonometric parallax from the original Hipparcos catalogue (ESA 1997) is $2.33 \pm 0.51$ mas. From a back-tracing of $\zeta$ Pup, Hoogerwerf et al. (2001) found that this star had a possible encounter with the cluster Trumpler 10 some 2 Myr ago provided that its dynamical distance was $d_{\text {dyn }}=250 \ldots 300$ pc. This was inconsistent with the Hipparcos distance of $430 \mathrm{pc}$, and also its absolute magnitude did not agree with the cluster isochrone. Our backtracing confirms the results from Hoogerwerf et al. (2001), giving Trumpler 10 as the host and $d_{\mathrm{dyn}}=300 \mathrm{pc}, t_{\mathrm{enc}}=2.5 \mathrm{Myr}$, $d_{\text {enc }}=0.9 \mathrm{pc}$, and $p_{\text {kin }}=0.94$. This result is consistent with the new Hipparcos parallax $(3.00 \pm 0.1$ mas $)$ from the re-reduction of Hipparcos data by van Leeuwen (2007). If we adopt the new Hipparcos distance of $333 \mathrm{pc}$, we find a solution with $t_{\mathrm{enc}}=$ $1.8 \mathrm{Myr}$ and $d_{\mathrm{enc}}=7.1 \mathrm{pc}$, which has only a slightly smaller probability $\left(p_{\text {kin }}=0.91\right)$.

In Table 1 we summarise the distances of $\zeta$ Pup from the various sources. Compared to the new Hipparcos benchmark, the old spectroscopic distance renders $\zeta$ Pup 1.34 mag too bright in absolute magnitude. According to our $d_{\text {dyn }}$ it would be 0.24 mag fainter than based on the revised Hipparcos distance. From a non-LTE analysis of the spectrum, Kudritzki et al. (1983) found that the effective temperature of $\zeta$ Pup is $T_{\text {eff }}=42000 \mathrm{~K}$ instead of $50000 \mathrm{~K}$ according to a spectral type of O4. This, together with the low $\log g=3.5$, means that $\zeta$ Pup is already away from the ZAMS.

Kudritzki et al. (1983) also determined the angular diameter of $\zeta$ Pup to be $\alpha=4.0 \times 10^{-4}$ arcsec. Together with an assumed distance of $(450 \pm 200) \mathrm{pc}$, this yielded a radius of $(19 \pm 8) R_{\odot}$ and a mass of $40 M_{\odot}$. Using the new Hipparcos parallax, we find a radius of $(14 \pm 0.4) R_{\odot}$ and a mass of $(22.5 \pm 1.3) M_{\odot}$. For its luminosity we find $\log L / L_{\odot}=5.74 \pm 0.02$. For the error calculation, only the mean error of the Hipparcos parallax is considered. The new Hipparcos parallax rules out the scenarios by van Rensbergen et al. (1996). They discussed an origin of $\zeta$ Pup in Vela R2, which would imply a present-day distance of the star of 700 to $800 \mathrm{pc}$. The alternative scenario, that $\zeta$ Pup originated as a field star and its runaway nature is due to a binary history, is now also ruled out, because this would lead to a present-day distance of between 400 and 800 pc (van Rensbergen et al. 1996).

To summarise, $\zeta$ Pup is closer, less massive, and less luminous than previously thought.

\subsection{HD 188209, HD 189957, HD 198846, HD 201345}

Although, at present, these stars are separated by hundreds of parsecs, they have common "relatives". We show their spatial distribution in Fig. 8 where $X, Y, Z$ are the Cartesian Galactic coordinates and $R G(\mathrm{pc})$ is the Galactocentric radius of a star or a cluster. The present-day location is shown in the left column 
Table 2. Results of the back-tracing calculations.

\begin{tabular}{|c|c|c|c|c|c|c|c|c|c|c|}
\hline HD & Sp. type & Cluster name & $\log t_{\mathrm{ocl}}$ & $\begin{array}{l}d_{\text {dyn }} \\
{[\mathrm{pc}]}\end{array}$ & $\begin{array}{r}t_{\mathrm{enc}} \\
{[\mathrm{Myr}]}\end{array}$ & $\begin{array}{l}d_{\text {enc }} \\
{[\mathrm{pc}]}\end{array}$ & $\begin{array}{c}\Delta V e l_{\text {enc }} \\
{\left[\mathrm{km} \mathrm{s}^{-1}\right]}\end{array}$ & $\begin{array}{c}M_{V}[\min , \max ] \\
{[\mathrm{mag}]}\end{array}$ & $p_{\text {kin }}$ & comments \\
\hline 1337 & O9.0 III & NGC 957 & 6.84 & 1911 & 8.5 & 3.9 & 75.7 & $-6.0[-6.4,-5.8]$ & 0.54 & \\
\hline 10125 & O9.7 II & IC 1590 & 6.84 & 2300 & 4.9 & 6.1 & 78.5 & $-5.3[-5.6,-5.0]$ & 0.81 & \\
\hline 12323 & $09.0 \mathrm{~V}$ & ASCC 9 & 6.79 & 2890 & 6.7 & 4.6 & 46.8 & $-4.0[-4.1,-3.5]$ & 0.97 & \\
\hline 12993 & $06.5 \mathrm{~V}$ & NGC 884 & 7.10 & 2074 & 4.3 & 0.3 & 61.2 & $-4.0[-4.2,-3.6]$ & 0.71 & \\
\hline 13022 & O9.5 II-III & NGC 869 & 7.28 & 1948 & 8.3 & 2.6 & 17.2 & $-4.3[-4.7,-4.1]$ & 0.88 & \\
\hline 13745 & O9.7 II & NGC 663 & 7.14 & 2035 & 4.7 & 2.5 & 50.5 & $-4.9[-5.2,-4.6]$ & 0.80 & \\
\hline 14434 & $\mathrm{O} 5.5 \mathrm{~V}$ & ASCC 9 & 6.79 & 3062 & 5.9 & 5.5 & 34.0 & $-5.2[-5.5,-4.9]$ & 0.81 & \\
\hline 14442 & $05.0 \mathrm{~V}$ & ASCC 9 & 6.79 & 2912 & 3.9 & 3.4 & 40.9 & $-5.2[-5.4,-4.8]$ & 0.94 & \\
\hline 14633 & $08.0 \mathrm{~V}$ & ASCC 8 & 6.76 & 2151 & 9.2 & 3.8 & 76.5 & $-4.5[-4.6,-4.0]$ & 0.89 & (1) \\
\hline 14947 & O5.0 I & ASCC 8 & 6.76 & 2177 & 1.2 & 8.8 & 32.1 & $-5.8[-6.1,-5.5]$ & 0.77 & \\
\hline 15137 & O9.5 II-III & NGC 957 & 6.84 & 1784 & 5.8 & 2.4 & 27.9 & $-4.3[-4.5,-3.9]$ & 0.92 & \\
\hline 16691 & O4.0 I & ASCC 9 & 6.79 & 2901 & 1.1 & 6.8 & 43.3 & $-5.8[-6.1,-5.5]$ & 0.55 & \\
\hline 17603 & O7.5 Ib & ASCC 8 & 6.76 & 2272 & 7.3 & 1.2 & 26.5 & $-6.1[-6.3,-5.7]$ & 0.97 & \\
\hline 39680 & $06.0 \mathrm{~V}$ & NGC 2169 & 6.89 & 1354 & 9.1 & 5.5 & 35.2 & $-3.6[-3.8,-3.2]$ & 0.84 & \\
\hline 41997 & $07.5 \mathrm{~V}$ & Collinder 89 & 7.50 & 723 & 2.8 & 2.4 & 44.4 & $-2.7[-3.0,-2.5]$ & 0.63 & \\
\hline 44811 & $07.0 \mathrm{~V}$ & NGC 2129 & 7.08 & 1582 & 9.5 & 8.9 & 18.3 & $-3.7[-4.0,-3.4]$ & 0.51 & \\
\hline 52266 & O9.0 IV & Collinder 106 & 6.74 & 1388 & 10.4 & 1.9 & 34.3 & $-4.3[-4.4,-3.8]$ & 0.55 & \\
\hline 52533 & $09.5 \mathrm{~V}$ & Collinder 106 & 6.74 & 1831 & 10.0 & 3.8 & 34.9 & $-4.2[-4.4,-3.7]$ & 0.86 & \\
\hline 57236 & $08.0 \mathrm{~V}$ & NGC 2414 & 6.94 & 2432 & 9.1 & 5.4 & 62.3 & $-4.6[-4.8,-4.2]$ & 0.84 & \\
\hline 66811 & O4.0 I & Trumpler 10 & 6.95 & 297 & 2.5 & 1.4 & 51.0 & $-5.2[-5.3,-4.7]$ & 0.94 & (1) \\
\hline 69464 & O6.5 Ib & ASCC 45 & 7.12 & 3001 & 0.1 & 0.8 & 11.4 & $-5.2[-5.6,-4.9]$ & 0.78 & (2) \\
\hline 75222 & O9.7 Iab & Collinder 205 & 7.03 & 1735 & 6.6 & 1.1 & 62.8 & $-5.6[-6.0,-5.4]$ & 0.97 & (1) \\
\hline 76968 & O9.7 Ib & ASCC 45 & 7.12 & 2682 & 10.6 & 4.1 & 81.2 & $-5.9[-6.5,-5.8]$ & 0.86 & \\
\hline 89137 & O9.5 III & Loden 306 & 6.76 & 2026 & 9.1 & 3.1 & 45.6 & $-4.3[-4.4,-3.8]$ & 0.91 & \\
\hline 91651 & $09.0 \mathrm{~V}$ & Trumpler 14 & 6.67 & 2720 & 1.6 & 2.3 & 46.3 & $-4.2[-4.3,-3.7]$ & 0.91 & \\
\hline 93632 & O5.0 III & Trumpler 14 & 6.67 & 2731 & 3.1 & 6.0 & 12.3 & $-5.6[-5.8,-5.1]$ & 0.74 & \\
\hline 94963 & O6.5 III & IC 2581 & 7.22 & 2504 & 8.7 & 6.5 & 27.8 & $-5.1[-5.6,-5.0]$ & 0.81 & \\
\hline 96917 & O8.5 Ib & Collinder 228 & 6.68 & 1991 & 3.1 & 1.9 & 55.5 & $-5.5[-5.7,-5.1]$ & 0.84 & \\
\hline 96946 & $06.0 \mathrm{~V}$ & vdBergh-Hagen 121 & 6.64 & 2738 & 4.1 & 7.8 & 49.2 & $-5.3[-5.4,-4.8]$ & 0.70 & \\
\hline 97848 & $08.0 \mathrm{~V}$ & NGC 3324 & 6.72 & 2329 & 4.0 & 8.8 & 48.0 & $-4.0[-4.1,-3.5]$ & 0.90 & \\
\hline 104565 & O9.7 Ia & ASCC 75 & 6.65 & 2839 & 4.7 & 3.2 & 148.0 & $-5.1[-5.2,-4.5]$ & 0.72 & \\
\hline 104649 & $09.5 \mathrm{~V}$ & NGC 3572 & 6.88 & 1948 & 5.8 & 2.2 & 40.2 & $-4.4[-4.6,-4.0]$ & 0.93 & \\
\hline 105056 & O9.7 Ia & Ruprecht 94 & 7.19 & 3304 & 9.0 & 3.8 & 47.4 & $-5.8[-6.4,-5.7]$ & 0.64 & (1) \\
\hline 105627 & O9.0 II-III & Loden 821 & 7.29 & 2885 & 6.5 & 7.5 & 70.8 & $-4.8[-5.3,-4.7]$ & 0.68 & \\
\hline 112244 & O8.5 Iab & Feinstein 1 & 6.97 & 1391 & 8.8 & 3.2 & 45.3 & $-6.0[-6.4,-5.8]$ & 0.83 & \\
\hline 116852 & O9.0 III & Trumpler 14 & 6.67 & 2475 & 6.0 & 2.7 & 179.6 & $-4.1[-4.2,-3.6]$ & 0.67 & (1) \\
\hline 117856 & O9.5 III & Loden 694 & 7.38 & 1750 & 10.0 & 5.2 & 17.4 & $-5.0[-5.5,-4.9]$ & 0.51 & \\
\hline 120521 & O8.0 Ib & Loden 821 & 7.29 & 2890 & 7.8 & 1.8 & 26.5 & $-5.0[-5.5,-4.8]$ & 0.80 & \\
\hline 120678 & O8.0 III & Loden 821 & 7.29 & 2864 & 5.9 & 1.5 & 39.8 & $-5.2[-5.8,-5.1]$ & 0.84 & \\
\hline 123008 & O9.7 Ib & Loden 821 & 7.29 & 2875 & 6.9 & 1.4 & 51.0 & $-5.1[-5.6,-5.0]$ & 0.66 & \\
\hline 123056 & $09.5 \mathrm{~V}$ & Loden 694 & 7.38 & 1577 & 10.1 & 3.1 & 28.5 & $-3.9[-4.3,-3.7]$ & 0.83 & \\
\hline 125206 & O9.5 IV & NGC 5606 & 6.84 & 2054 & 7.2 & 3.6 & 41.1 & $-5.2[-5.5,-4.9]$ & 0.68 & \\
\hline 135240 & O7.5 III & ASCC 79 & 6.86 & 809 & 2.2 & 0.6 & 5.2 & $-5.0[-5.3,-4.7]$ & 0.73 & (3) \\
\hline 135591 & O7.5 III & ASCC 79 & 6.86 & 796 & 0.3 & 1.4 & 17.1 & $-4.6[-4.8,-4.2]$ & 0.74 & (3) \\
\hline 148546 & O9.0 Ia & ASCC 88 & 7.17 & 1453 & 8.8 & 5.7 & 55.4 & $-4.7[-5.0,-4.4]$ & 0.73 & (1) \\
\hline 153426 & O9.0 II-III & Hogg 22 & 6.70 & 1694 & 8.1 & 9.9 & 55.4 & $-4.9[-5.1,-4.5]$ & 0.91 & \\
\hline 153919 & O6.5 Ia & NGC 6231 & 6.81 & 1034 & 5.1 & 1.1 & 48.8 & $-5.1[-5.4,-4.7]$ & 0.82 & \\
\hline 154368 & O9.5 Iab & Sco OB4 & 6.82 & 1082 & 4.7 & 5.3 & 11.2 & $-6.3[-6.6,-6.0]$ & 0.65 & \\
\hline 154643 & O9.5 III & ASCC 88 & 7.17 & 1908 & 1.4 & 2.3 & 20.3 & $-5.6[-6.1,-5.5]$ & 0.85 & \\
\hline 154811 & O9.7 Iab & vdBergh-Hagen 205 & 7.12 & 2019 & 8.2 & 2.7 & 35.1 & $-6.2[-6.8,-6.2]$ & 0.96 & \\
\hline 156212 & O9.7 Iab & Trumpler 28 & 6.89 & 992 & 7.0 & 4.0 & 53.5 & $-4.5[-4.7,-4.1]$ & 0.93 & (1) \\
\hline 157857 & O6.5 III & NGC 6611 & 6.72 & 1854 & 3.8 & 2.6 & 113.5 & $-4.9[-5.1,-4.5]$ & 0.68 & (1) \\
\hline 158186 & $09.5 \mathrm{~V}$ & Sco OB4 & 6.82 & 1254 & 5.6 & 1.2 & 30.3 & $-4.4[-4.6,-4.0]$ & 0.82 & \\
\hline 161853 & $08.0 \mathrm{~V}$ & Trumpler 28 & 6.89 & 1241 & 5.0 & 2.6 & 25.4 & $-4.1[-4.3,-3.7]$ & 0.68 & \\
\hline 166734 & $\mathrm{O} 7.0 \mathrm{Ib}$ & NGC 6604 & 6.64 & 1661 & 3.0 & 2.5 & 22.4 & $-6.7[-7.0,-6.4]$ & 0.65 & \\
\hline 169515 & O9.7 Ib & Markarian 38 & 6.95 & 1794 & 8.9 & 4.3 & 37.7 & $-6.1[-6.5,-5.9]$ & 0.54 & \\
\hline 169582 & O6.0 I & NGC 6604 & 6.64 & 1565 & 5.5 & 3.2 & 28.5 & $-4.9[-5.0,-4.4]$ & 0.57 & \\
\hline 171589 & O7.0 II & NGC 6618 & 6.78 & 1934 & 3.3 & 1.5 & 63.2 & $-4.9[-5.1,-4.5]$ & 0.96 & \\
\hline 175754 & O8.0 II & ASCC 93 & 7.22 & 2586 & 10.3 & 4.9 & 64.8 & $-5.3[-5.8,-5.2]$ & 0.47 & \\
\hline 175876 & O6.5 III & NGC 6618 & 6.78 & 1922 & 5.5 & 4.8 & 61.7 & $-5.0[-5.2,-4.5]$ & 0.88 & \\
\hline 188001 & O7.5 Ia & Roslund 2 & 6.89 & 2022 & 2.7 & 2.5 & 70.3 & $-6.0[-6.4,-5.7]$ & 0.79 & (1) \\
\hline 188209 & O9.5 Iab & NGC 6871 & 6.99 & 1705 & 10.9 & 7.3 & 33.7 & $-5.9[-6.4,-5.7]$ & 0.57 & \\
\hline 189957 & O9.5 III & Cyg OB2 & 6.72 & 1858 & 5.9 & 3.1 & 67.7 & $-4.4[-4.6,-4.0]$ & 0.87 & (1) \\
\hline 192281 & $05.0 \mathrm{~V}$ & NGC 6913 & 7.12 & 1081 & 2.4 & 1.2 & 35.5 & $-4.5[-4.8,-4.2]$ & 0.80 & \\
\hline 195592 & O9.7 Ia & NGC 6913 & 7.12 & 1132 & 2.9 & 6.3 & 40.3 & $-6.3[-6.9,-6.3]$ & 0.79 & \\
\hline
\end{tabular}


Table 2. continued

\begin{tabular}{lllrrrrrrrr}
\hline \hline 198846 & O9.0 V & Cyg OB2 & 6.72 & 1427 & 1.9 & 3.7 & 106.6 & $-4.1[-4.3,-3.7]$ & 0.81 & $(1)$ \\
201345 & O9.0 V & Cyg OB2 & 6.72 & 1739 & 5.8 & 1.5 & 65.6 & $-4.0[-4.1,-3.5]$ & 0.99 & \\
203064 & O7.5 III & Collinder 419 & 6.85 & 631 & 6.5 & 1.3 & 26.9 & $-4.8[-5.0,-4.4]$ & 0.87 & $(1)$ \\
218915 & O9.5 Iab & ASCC 120 & 7.08 & 2369 & 5.8 & 4.3 & 54.7 & $-5.4[-5.8,-5.1]$ & 0.72 & $(1)$ \\
303492 & O9.0 Ia & Loden 153 & 6.74 & 2714 & 1.5 & 2.6 & 79.3 & $-5.8[-6.1,-5.5]$ & 0.71 & \\
305523 & O9.0 II & ASCC 65 & 7.09 & 3532 & 7.5 & 6.3 & 27.8 & $-5.3[-5.7,-5.1]$ & 0.81 & \\
305532 & O6.0 V & ASCC 65 & 7.09 & 3448 & 3.6 & 6.7 & 58.0 & $-4.3[-4.6,-4.0]$ & 0.80 & \\
305539 & O7.0 V & Trumpler 14 & 6.67 & 2752 & 1.4 & 1.3 & 21.3 & $-4.1[-4.2,-3.6]$ & 0.83 & \\
\hline
\end{tabular}

Comments: (1) runaway O-stars according to Sota et al. (2007); (2) member of the ASCC 45 cluster according to Kharchenko et al. (2005b); (3) member of the ASCC 79 cluster according to Kharchenko et al. (2005b).

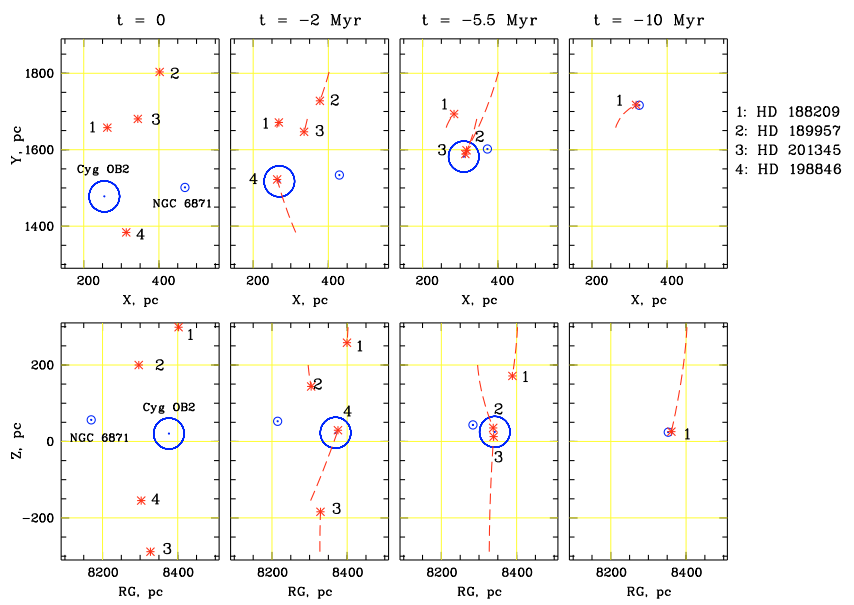

Fig. 8. Spatial distribution of the O-stars (1: HD 188209, 2: HD 189957, 3: HD 201345, 4: HD 198846), of the open cluster NGC 6871, and of the association Cyg OB 2. The upper panels are for the $(X, Y)$-plane, the lower panels show the distribution in the $(R G, Z)$-plane, where $\mathrm{RG}$ is the Galactocentric radius. Asterisks mark the location of stars, the circles represent the clusters; their radii are defined as the present-day tidal radii determined in Piskunov et al. (2008a). The left columns show the location at present; the second, third, and forth columns show the locations $2 \mathrm{Myr}$, $5.5 \mathrm{Myr}$, and $10 \mathrm{Myr}$ ago, respectively. The dashed curves in these panels delineate the stellar orbits computed with the back-tracing procedure from the present to the corresponding time $t$ indicated on the top. Note that Cyg OB 2 is younger than $10 \mathrm{Myr}$, and therefore does not appear in the right-most column.

$(t=0)$, the right column $(t=-10 \mathrm{Myr})$ displays the same region $10 \mathrm{Myr}$ ago. At that time the open cluster NGC $6871 \mathrm{had}$ formed (its age is dated $\log t=6.99$ in Kharchenko et al. 2005a). Just before, at $t_{\mathrm{enc}}=10.9 \mathrm{Myr}$, the O-star HD 188209 escaped from this region with a relative velocity of about $35 \mathrm{~km} \mathrm{~s}^{-1}$, away from the Galactic plane, and towards the North pole. A few million years later $(t=-7.7 \mathrm{Myr}$, not shown in Fig. 8), NGC 6871 passed at about 35 pc of the region where the association Cyg OB 2 came into being at $t \approx-5.5 \mathrm{Myr}$. Kharchenko et al. (2005a) give $\log t=6.72$ for Cyg OB 2. Immediately before, two O-stars were ejected from this region, HD 189957 $\left(t_{\mathrm{enc}}=5.9 \mathrm{Myr}\right)$ and HD $201345\left(t_{\mathrm{enc}}=5.8 \mathrm{Myr}\right)$. HD 189957 started in the direction of the North pole with a relative velocity of about $70 \mathrm{~km} \mathrm{~s}^{-1}$ whereas HD 201345 moved to the South pole with about the same relative velocity. About 3.5 Myr later ( $t=-2$ Myr) the O-star HD 198846 was ejected from Cyg OB 2 with a relative velocity higher than $100 \mathrm{~km} \mathrm{~s}^{-1}$ in the direction to the South pole, too.

As we note in Sect. 3 and show in Fig. 2, there is another acceptable solution for HD 201345, although with a slightly lower kinematic probability, suggesting an ejection from NGC 6871 about 6.5 Myr ago. Also for HD 189957, a second acceptable solution was possible (an ejection from NGC 6871 with the parameters $\left.d_{\text {dyn }}=1964 \mathrm{pc}, t_{\mathrm{enc}}=7.1 \mathrm{Myr}, d_{\mathrm{enc}}=3.5 \mathrm{pc}, p_{\text {kin }}=0.84\right)$. Since the time of their closest approach with NGC 6871 is comparable with the time when NGC 6871 passed the birth place of Cyg OB 2, one may suspect that this event was the reason for the ejection of HD 201345 and HD 189957 from a region in between NGC 6871 and Cyg OB 2.

\subsection{O-stars at large distances from the Galactic plane}

The majority of O-stars in our sample is located within 200 pc from the Galactic plane. However, there are five stars with a present-day location at $|Z|>400 \mathrm{pc}$. These stars either had relative velocities at the moment of encounter (ejection) higher than $100 \mathrm{~km} \mathrm{~s}^{-1}$ (HD 116852, HD 157857) or they left their cluster counterparts more than 9 Myr ago (HD 14633, HD 105056, HD 175754). Below, we consider two examples, HD 116852 and HD 14633, which have the largest distances from the Galactic plane. These cases are also interesting as their counterparts were clusters hosting other field O-stars, too.

\subsubsection{HD 116852 and Trumpler 14}

Judging from the spectroscopic distance and high galactic latitude of HD 116852, one can expect that this star is located at a relatively large distance from the Galactic plane. Indeed, from back-tracing we obtain $Z \approx-690$ pc for the present-day location of HD 116852. This star left its birth-place in a protocluster of Trumpler 14 with a relative velocity of $\approx 180 \mathrm{~km} \mathrm{~s}^{-1}$ about $6 \mathrm{Myr}$ ago, i.e. just before the cluster was formed. Kharchenko et al. (2005a) list $\log t=6.67$ for Trumpler 14. The location of the star and of the protocluster at that time was at $Z \approx-50 \mathrm{pc}$. During the next $6 \mathrm{Myr}$, the cluster moved by only $60 \mathrm{pc}$, whereas HD 116852 moved more than $1 \mathrm{kpc}$. Just $1.5 \mathrm{Myr}$ after its birth, Trumpler 14 lost another O-star, HD 93652, and about 1.5 Myr later, HD 91651 and HD 305539 left the cluster. Presently, HD 93652, HD 91651, and HD 305539 are located about $40 \mathrm{pc}, 75 \mathrm{pc}$, and $30 \mathrm{pc}$ away from Trumpler 14, respectively. According to Mel'nik \& Efremov (1995), there is an OBassociation Car $1 \mathrm{~F}$ at about $65 \mathrm{pc}$ from Trumpler 14. Probably, this neighbourhood has had impact on the fate of Trumpler 14.

\subsubsection{HD 14633 and ASCC 8}

Though presently HD 14633 is at $Z \approx-670$ pc, about 9 Myr ago this star was located near the Galactic plane between the clusters NGC 869 (h Per) and NGC 884 ( $\chi$ Per), i.e. within a region well known as the then very active star formation region Per OB 1. About 3.5 Myr later this place was also the birth-place 

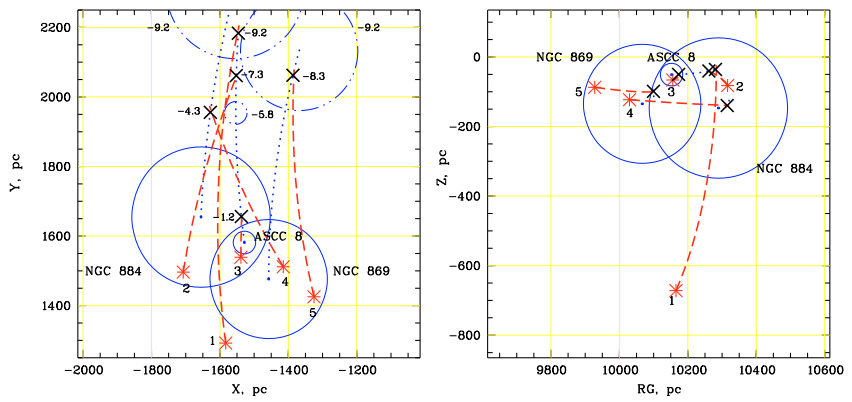

Fig. 9. Spatial distribution of the field O-stars (1: HD 14633, 2: HD 17603, 3: HD 14947, 4: HD 12993, 5: HD 13022), and of the open clusters NGC 869 , NGC 884 , ASCC 8 . The left panel is for the $(X, Y)$ plane, the right panel shows the distribution in the $(R G, Z)$-plane, $\mathrm{RG}$ is the Galactocentric radius. Asterisks mark the present-day location of the stars, the circles are for the clusters: solid curves for the present-day location and dash-dot-dot-curves for the past. Their radii are defined by the present-day tidal radii determined in Piskunov et al. (2008a). The dashed and dotted curves show the orbits of the stars and clusters, respectively, computed with the back-tracing procedure. The crosses mark the places where the stars encountered their hosts. Negative numbers in the left panel express the time in Myr when the corresponding events happened (in order not to overload the figure, the numbers are omitted in the right panel).

of an open cluster, ASCC $8(\log t=6.76)$. In Fig. 9 we show the location of these three clusters (NGC 869, NGC 884, ASCC 8) as it is now and as it was in the past.

According to the tidal radii determined in Piskunov et al. (2008a), NGC 869 and NGC 884 are the largest clusters in the whole COCD cluster sample. Moreover, their tidal spheres are overlapping, and some concentration of stars above the background is observed in this overlapping zone. Based on common proper motions, an open cluster (ASCC 8) was identified here (Kharchenko et al. 2005b), with an age of about 5.8 Myr. Such a location of a cluster does not make a long life very likely. From the back-tracing computations, three O-stars marked as field in the GOSV2 came from the region now occupied by ASCC 8: HD 14947 - 1.2 Myr ago, HD 17603 - 7.3 Myr ago, and HD 14633 - 9.2 Myr ago. Two other field stars, HD 12993 and HD 13022, originated in NGC 884 and NGC 869, respectively. Note that the final solution for a given star and its host is selected from all acceptable solutions $\left(d_{\mathrm{enc}}<10 \mathrm{pc}\right)$ to have the smallest encounter distance $d_{\text {enc }}$ at the highest kinematic probability $p_{\text {kin. }}$. This approach is justified if possible hosts (i.e. open clusters) are separated by distances that are considerably larger than their sizes. This is the usual case. However, the example considered presents an exception to the rule. Here the distances between cluster centres are comparable to their tidal radii. Therefore, solutions with $d_{\text {enc }}>10$ pc can be considered, too. For example, HD 12993 could have been ejected from NGC 869 about $0.8 \mathrm{Myr}$ ago when the star was $\approx 50 \mathrm{pc}$ away from the cluster centre. In this special case, we prefer a more general statement: the observational data for these five field O-stars are consistent with the claim that they originated in Per OB 1.

\section{Stars without solutions}

For 73 out of $105 \mathrm{O}$-stars with assignments as field stars in the GOSV2 we could trace back their origins and found the corresponding host clusters or protoclusters. Twelve stars could not be treated by our method, because no radial velocities were available for them. For another twenty, we could not find a solution, i.e. they could not be associated with any of the clusters of our sample in the past 10 million years. This negative finding, however, should not be interpreted as a proof that these $\mathrm{O}$-stars have formed in isolation.

We could not find a parameter or parameters that distinguish these stars from the other field O-stars which had an acceptable solution. Both groups cover a similar range of apparent magnitudes, and they are comparable in the distribution of the mean errors of their kinematic components. Of course, one cannot exclude that a few of them have a true distance modulus differing from the spectroscopic estimates by more than two magnitudes, and/or their true velocity components are outside the intervals checked with the backward procedure. Nevertheless, a more important aspect seems to be that our sample of potential host clusters is not complete for associations and very young (embedded) clusters, as we stressed in Sect. 2.1. Provided that this assumption is correct, one would expect about $20 \%$ of field O-stars to have their origins in these kinds of objects. To answer the question with certainty, however, more accurate data on the distances and kinematics of associations and embedded clusters are required.

\section{Summary}

In this paper we have followed the dynamical history of O-stars that left the groups in which they originated. We cannot conclude on the physical mechanisms that underlie these events.

For 73 out of $93 \mathrm{O}$-stars considered, we found acceptable solutions indicating that the present-day data are consistent with the assumption that these O-stars were ejected from young open clusters or protoclusters over the past 10 Myr. The GOSV2 catalogue counts $370 \mathrm{O}$-stars, for 265 of which the origin is given in that catalogue. We were able to add 73 more cases to the list. For 32 stars (or 9\%) we could not prove an origin in a group.

In this paper we have dealt with aspects of the early phases in the life of open clusters. O-stars are best suited as tracers of this early-phase evolution because of their short life-time. In our sample of 161 young open clusters (age < $30 \mathrm{Myr}$ ) from the COCD there are 55 (or $\approx 35 \%$ ) that have O-stars as members (Kharchenko et al. 2005a,b); 23 of these have already lost one or more O-stars in their history. Another 24 (or 15\%) of the COCD clusters have been related to O-stars in the past 10 Myrs, but do not contain O-stars at present. For 82 (51\%) young clusters we cannot prove a relationship with presently living O-stars. Either their most massive member is a main sequence star of spectral type later than $\mathrm{O}$, or it is a former $\mathrm{O}$-star that already has evolved. Of the 47 clusters that have lost at least one O-star, we find 14 that are so young that the O-star and cluster already should have separated in the protocluster phase.

Summing up the statistics above, the following picture emerges. Fifty percent of the clusters able to survive the infantmortality phase are so massive that they contain or contained $\mathrm{O}$-stars. These O-stars have not been able to destroy the cluster. This, in parts, answers the question asked by Lada \& Lada (2003) in the affirmative: do the progenitors of bound open clusters ever contain O-stars?

The fact, that we could not trace back $9 \%$ of all O-stars from the GOSV2 does not necessarily mean that "isolated O-star formation" is possible. Their known astrophysical data (distance, velocity) may be incorrect or our list of possible host candidates may be incomplete. On the other hand, we can interpret our result - no solution for 20 out of 93 stars - as follows: there is an upper bound of slightly more than $20 \%$ of O-stars 
that could have destroyed the group of other objects with which they formed.

It has been shown by Piskunov et al. (2008b) that classical (gravitationally bound) open clusters in the Milky Way evolve due to stellar and dynamical evolution as well as due to external perturbations. They are losing stellar mass during their life-time at an average rate of 3 to $14 M_{\odot} / \mathrm{Myr}$. In this paper we determined the mass loss rate of young open clusters due to O-stars alone to be 3 to $5 M_{\odot} / \mathrm{Myr}$ in the first few million years of their existence.

As a by-product, we find new distances and absolute magnitudes for $73 \mathrm{O}$-stars. These indicate that the calibration of $\mathrm{ab}$ solute magnitudes of $\mathrm{O}$-stars should be revised. Their absolute magnitudes are systematically fainter by about 0.3 to 0.8 mag compared to the calibrations by Garmany \& Stencel (1992), Walborn (1972), or Schmidt-Kaler (1982). This would be consistent with the re-calibration of the absolute magnitudes of early B-type stars by Kaltcheva (1999) using Hipparcos trigonometric parallaxes.

We have also shown that $\zeta$ Pup, the closest O-star from the Sun, left the young open cluster Trumpler 10 some 2.5 Myr ago. Its present-day distance from the Sun of $300 \mathrm{pc}$ is compatible to the new Hipparcos distance from van Leeuwen (2007). This implies a radius of $14 R_{\odot}$, a mass of $22.5 M_{\odot}$, and a luminosity of $\log L / L_{\odot}$ of 5.74 for $\zeta$ Pup, i.e. the values are considerably smaller than assumed before.

Acknowledgements. We are grateful to Nina Kharchenko, Anatoly Piskunov and Hans Zinnecker for fruitful discussions on massive star formation and young open clusters.

\section{References}

Blaauw, A. 1961, Bull. Astron. Inst. Netherlands, 15, 265

Conti, P. S., Garmany, C. D., de Lore, C., \& Vanbeveren, D. 1983, ApJ, 274, 302 de Wit, W. J., Testi, L., Palla, F., Vanzi, L., \& Zinnecker, H. 2004, A\&A, 425, 937

de Wit, W. J., Testi, L., Palla, F., \& Zinnecker, H. 2005, A\&A, 437, 247

de Zeeuw, P. T., Hoogerwerf, R., de Bruijne, J. H. J., Brown, A. G. A., \& Blaauw, A. 1999, AJ, 117, 354

ESA 1997, VizieR Online Data Catalog, 1239, 0

Fuchs, B., Breitschwerdt, D., de Avillez, M. A., Dettbarn, C., \& Flynn, C. 2006, MNRAS, 373, 993

Garmany, C. D., \& Stencel, R. E. 1992, A\&AS, 94, 211
Gies, D. R. 1987, ApJS, 64, 545

Girardi, L., Bertelli, G., Bressan, A., et al. 2002, A\&A, 391, 195

Hoogerwerf, R., de Bruijne, J. H. J., \& de Zeeuw, P. T. 2001, A\&A, 365, 49

Houk, N., \& Swift, C. 1999, Michigan catalogue of two-dimensional spectral types for the HD Stars, Vol. 5 (Ann Arbor, Michigan: Department of Astronomy, University of Michigan)

Kaltcheva, N. 1999, in, Harmonizing Cosmic Distance Scales in a PostHIPPARCOS Era, ed. D. Egret, \& A. Heck, ASP Conf. Ser., 167, 263

Kharchenko, N. V. 2001, Kinematics and Physics of Celestial Bodies, 17, 409

Kharchenko, N. V., Piskunov, A. E., Röser, S., Schilbach, E., \& Scholz, R.-D. 2004, Astron. Nachr., 325, 740

Kharchenko, N. V., Piskunov, A. E., Röser, S., Schilbach, E., \& Scholz, R.-D. 2005a, A\&A, 438, 1163

Kharchenko, N. V., Piskunov, A. E., Röser, S., Schilbach, E., \& Scholz, R.-D. 2005b, A\&A, 440, 403

Kharchenko, N. V., Scholz, R.-D., Piskunov, A. E., Röser, S., \& Schilbach, E. 2007, Astron. Nachr., 328, 889

Kroupa, P., \& Boily, C. M. 2002, MNRAS, 336, 1188

Kudritzki, R. P., Simon, K. P., \& Hamann, W.-R. 1983, A\&A, 118, 245

Lada, C. J., \& Lada, E. A. 2003, ARA\&A, 41, 57

Lindblad, B. 1959, Handbuch der Physik, 53, 21

Maíz-Apellániz, J., Walborn, N. R., Galué, H. Á., \& Wei, L. H. 2004, ApJS, 151, 103

Mel'nik, A. M., \& Efremov, Y. N. 1995, Astron. Lett., 21, 10

Neckel, T., Klare, G., \& Sarcander, M. 1980, A\&AS, 42, 251

Piskunov, A. E., Kharchenko, N. V., Röser, S., Schilbach, E., \& Scholz, R.-D. 2006, A\&A, 445, 545

Piskunov, A. E., Schilbach, E., Kharchenko, N. V., Röser, S., \& Scholz, R.-D. 2008a, A\&A, 477, 165

Piskunov, A. E., Kharchenko, N. V., Schilbach, E., et al. 2008b, A\&A, 487, 557

Röser, S., Schilbach, E., Schwan, H., et al. 2008, A\&A, 488, 401

Schilbach, E., Kharchenko, N. V., Piskunov, A. E., Röser, S., \& Scholz, R.-D. 2006, A\&A, 456, 523

Schmidt-Kaler, T. 1982, Landolt-Börnstein: Numerical Data and Functional Relationships in Science and Technology, ed. L. H. Aller, I. Appenzeller, B. Baschek, et al. (Berlin, Heidelberg, New York: Springer Verlag), New Series IV 2b, 1

Sota, A., Maíz Apellániz, J., Walborn, N. R., \& Shida, R. Y. 2007, [arXiv: astro-ph/0703005]

van Leeuwen, F. 2007, Hipparcos, the New Reduction of the Raw Data Astrophys. Space Sci. Libr., 350 (Springer Dordrecht)

van Rensbergen, W., Vanbeveren, D., \& de Loore, C. 1996, A\&A, 305, 825

Walborn, N. R. 1972, AJ, 77, 312

Walborn, N. R. 2002, AJ, 124, 507

Zinnecker, H. 2003, in, A Massive Star Odyssey: From Main Sequence to Supernova, ed. K. van der Hucht, A. Herrero, \& C. Esteban, IAU Symposium, 21280

Zinnecker, H., \& Yorke, H. W. 2007, ARA\&A, 45, 481 